\title{
JOSÉ ZARAGOZÁ ANTICH Y LA ESCUELA DE ORIENTACIÓN Y APROVECHAMIENTO DEL EXCMO. AYUNTAMIENTO DE VALENCIA (1940-1971), POSTERIOR INSTITUTO MUNICIPAL DE ORIENTACIÓN (1971-1981)
}

\author{
JOSÉ ZARAGOZÁ ANTICH AND THE «ESCUELA DE ORIENTACIÓN \\ Y APROVECHAMIENTO DEL EXCMO. AYUNTAMIENTO OF VALENCIA» \\ (1940-1971) LATER «INSTITUTO MUNICIPAL DE ORIENTACIÓN» (1971-1981)
}

\author{
José A. Benavent* \\ Universidad de Valencia
}

\section{RESUMEN}

La historia de la Orientación Psicopedagógica en España está en proceso de elaboración. Este artículo, resultado de una cuidadosa investigación histórica, nos muestra el papel desempeñado por D. José Zaragozá Antich en la creación, desarrollo, organización, consolidación y funcionamiento de la Escuela Especial de Orientación y Aprovechamiento del Excmo. Ayuntamiento de Valencia (1940-1971) y su posterior transformación en Instituto Municipal de Orientación (1971-1981).

Palabras clave: Historia de la Orientación Psicopedagógica en España. José Zaragozá Antich. Escuela Especial de Orientación y Aprovechamiento del Excmo. Ayuntamiento de Valencia. Instituto Municipal de Orientación de Valencia.

\footnotetext{
ABSTRACT

The history of Spanish Educational and Vocational Guidance is now being made. This article, outcome of a careful historical research, sketches the role played by Mr. José Zaragozá Antich in the foundation, evolution, organization, strengthening and work of the «Escuela Especial de Orientación y

* Perfil académico y profesional del Autor: Doctor en Filosofía y Letras (Pedagogía) y Master in Educational and Vocational Guidance. Profesor Titular de Universidad del Departamento de Didáctica y Organización Escolar de la Facultad de Filosofía y Ciencias de la Educación de la Universidad de Valencia. Líneas de investigación: Historia de la Orientación psicopedagógica en España; programas y metodología de la orientación y la toma de decisiones en la Educación Secundaria; análisis, evaluación y prospectiva de la Orientación psicopedagógica en la actual Reforma educativa.
} 
Aprovechamiento del Excmo. Ayuntamiento de Valencia» and their later conversion into «Instituto Municipal de Orientación» of Valencia (Spain).

Key words: History of Spanish Educational and Vocational Guidance. José Zaragozá Antich. Escuela Especial de Orientación y Aprovechamiento del Excmo. Ayuntamiento de Valencia. Instituto Municipal de Orientación de Valencia (Spain).

\section{La escuela de orientación y aprovechamiento del Excmo. Ayuntamiento de Valencia (1940-1971)}

\section{Creación}

La Escuela de Orientación y Aprovechamiento del Excmo. Ayuntamiento de Valencia (EEOA) nace de la propuesta de creación de una institución para la selección de niños bien dotados que hace Vicente García Llácer, Director de la Escuela Aneja de la Escuela Normal del Magisterio, al Alcalde de Valencia, el Barón de Cárcer, a finales de 1939. La propuesta es estudiada por el Dr. Barcia Goyanes, Presidente de la Comisión Municipal de Educación, quien solicita se amplíe incluyendo también a los alumnos «anormales o retrasados» y pide a García Llácer que redacte y presente un anteproyecto. Al Alcalde le gusta el anteproyecto, que será aprobado por el Pleno del Ayuntamiento en sesión extraordinaria de 31 de enero de 1940. En abril se le dotó de presupuesto y de personal y entró en funcionamiento el 1 de octubre de dicho año. Desde su aprobación, hasta la apertura de la Escuela se realizaron trabajos previos para suministrar los materiales psicopedagógicos imprescindibles para desarrollar los objetivos formulados en el anteproyecto, dar publicidad de su creación, realizar la selección de sus futuros alumnos y buscar unos locales adecuados para su ubicación (Memoria, 1940).

La EEOA se instaló en el Palacio de Polavieja en la plaza del Músico Gomis nº 6, edificio antiguo con patio, situándose las aulas de clase, dos para niños y otras dos para niñas en el primer piso, en alas opuestas y separadas por el despacho del director y el gabinete destinado a Laboratorio psicotécnico; en la planta baja se montaron los talleres y otras dependencias. En estos locales permaneció la Escuela hasta 1947, fecha en que se trasladó a un edificio mas amplio situado en la calle Salvador Giner $n^{\circ} 5$, donde permaneció hasta su transformación en Instituto Municipal de Orientación (IMO) en el curso 1970-71.

\section{Principios doctrinales}

Los principios ideológicos que animaron a García Llácer en la puesta en marcha de la EEOA se recogen en la siguiente cita que extraemos de la «Memoria» (1940) y que concuerdan plenamente con el espíritu del «nacional-catolicismo» de la postguerra:

«El sentido de servicio cristiano de este Centro, quiere llevar a término -con el estudio y medios técnicos puestos a su alcance- haciendo realidad, la filosofía vivista de ayuda y convivencia que se traduce en caridad, palabra síntesis de los valores de la filosofía del Nuevo Estado, que trata de sentar la jerarquía en la selección de los mejores y que procura adaptar el medio a los menguados de disposiciones» (Memoria, 1940, p. 4). 
Por otra parte, en una reseña reportaje sobre el funcionamiento de la EEOA durante sus primeros años, se dice: «La Escuela tiene capellán que orienta y dirige su vida espiritual. Los niños oyen misa diariamente. De acuerdo con el Frente de Juventudes, para realizar la educación política se cuenta con Instructor e Instructora de gimnasia e instrucción premilitar. También se desarrolla un amplio programa de nacional-sindicalismo» (Anónimo, 1943, p. 102).

Los principios científicos que fundamentaron la Orientación psicopedagógica en la EEOA parten de los planteamientos personalistas de la psicología escolástica, fundamentados en el culturalismo evolucionista y la axiología de Spranger (1935) y en el concepto de vocación de Gregorio Marañón (1936). García Llácer entiende, que cualquier fórmula que se emplee para orientar al hombre, «... siempre tendrá que ser resuelta frente al hombre completo, portador de valores eternos, en quien, necesariamente habrá de decidirse una general y efectiva disposición a desarrollarse preferentemente en determinada dirección, mejor que en otra, gracias a una capacidad física o funcional, intelectual y afectiva que es lo que crea la vocación» (García Llácer, 1943, p. 88). Por tanto, la Orientación tiene por objetivo descubrir, despertar y encauzar la auténtica vocación de cada hombre.

Este complejo proceso, lo sintetiza García Llácer en las siguientes proposiciones (García Llácer, 1943, p. 89):

«a) Cada hombre tiene su vocación».

«b) Como la vocación no siempre aflora, se precisa despertar el ímpetu vocacional».

«c) Realizar la vocación es vivir, en plenitud, el sentido de la profesión. Vocaciones erradas, si no se superan y adaptan, vegetan en la mediocridad o se derrumban en la neurosis. Debemos eliminar este riesgo por exigencia de la cultura y hasta por imperativo de caridad».

«d) La tarea de descubrir y orientar una vocación exige el adentrarse en la indivisible y total personalidad del candidato para conocerla, estimarla e interpretar su actitud ante los valores objetivos y subjetivos de toda profesión».

«e) En el hombre, la inteligencia global es una. Su rango establece la ecuación hombreprofesión. Pero matizan su jerarquía las tendencias, la capacidad funcional y la constelación del medio».

«f) Conocer los factores que se juegan en el punto anterior y conjugarlos debidamente es estar en condiciones de dar un consejo orientador coincidente con la auténtica vocación del individuo».

Para la medida psicotécnica de las aptitudes García Llácer sigue la corriente psicotécnica europea representada por Claparède (1924 y 1927) y la tradición del Institut d'Orientació Professional de Barcelona, del que procedía José Zaragozá y que visitó personalmente en 1940, sin citar a Mira y López, en aquellos momentos en el exilio y condenado por el régimen franquista, pero si que utilizará sus pruebas y baterías y el libro de Chleusebairgue, Orientación Profesional (1934), que recoge y sintetiza la doctrina y la metodología del extinto Institut Psicotècnic de la Generalitat de Catalunya.

Partiendo de los anteriores principios doctrinales y científicos, los fines que se propone la EEOA y las actividades que ha de desarrollar están perfectamente definidos en sus Bases fundacionales y obedecen a un triple propósito (Memoria, 1940, pp. 4-5):

«a) Seleccionar, atender y orientar profesionalmente a los niños bien dotados de la ciudad. 
b) Ejercer una terapéutica pedagógica en los niños intelectualmente deficientes, previamente seleccionados por la Escuela y

c) Investigar el desarrollo psicológico infantil, adaptar y elaborar instrumentos y métodos para la medida de las aptitudes.»

En la remodelación de las Bases de 1944 se asumen los tres fine fundacionales anteriormente citados y se añade uno nuevo:

«d) Ejercer una tutela y paternal vigilancia sobre los estudiantes y becarios del Ayuntamiento, durante el tiempo de estudios, tanto en el ámbito de la Segunda Enseñanza, como en la Enseñanza Superior y Profesional.»

\section{Evolución}

La vida de la EEOA se prolongó a lo largo de las décadas de los años 40, 50 y 60, del siglo pasado con distintos ritmos funcionales y cambios en la dirección de la misma, aunque sus Bases permanecieron sin modificación alguna desde 1944 hasta su reconversión en 1971 en Instituto Municipal de Orientación (IMO). Como IMO perduró hasta 1981 (jubilación de José Zaragozá), año en que se transforma por acuerdo del Pleno Municipal del Ayuntamiento de Valencia en Instituto Municipal de Educación (IME).

Durante sus 31 años de funcionamiento como EEOA podemos distinguir cuatro períodos claramente diferenciados y que se corresponden con los cambios que se producen en la dirección de la misma:

$1^{\circ}$ Período: Desde su fundación en 1940 hasta la finalización del curso académico 1947-48. Durante este primer período podemos distinguir dos etapas: a) Etapa fundacional, desde 1940 hasta junio de 1944 en que se aprueba la remodelación de sus Bases y b) Etapa de consolidación, desde principios del curso académico 1944-45 hasta el final del curso 1947-48 en que Vicente García Llácer deja el cargo de Director.

$2^{\circ}$ Período: Desde principios del curso 1948-49, en que José Zaragozá Antich (hasta entonces Maestro técnico auxiliar de Laboratorio), es nombrado Director de la Escuela, hasta el final del curso 1956-57, momento en que cesó de su cargo de Director y marchó a Guatemala como Jefe de una misión educativa de la UNESCO. José Zaragozá permanecería en América desempeñando distintas misiones hasta 1968 en que regresó de nuevo a España.

$3^{\circ}$ Período: Desde principios del curso 1957-58 hasta finales del curso 1967-68. Para sustituir a Zaragozá en la dirección de la Escuela se nombra a Felipe Gaspar Olmos.

$4^{\circ}$ Período: Desde principios del curso académico 1968-69 hasta el final del curso 1970-71, momento en que la EEOA se transforma en IMO. Durante este período la dirección volvió a recaer en José Zaragozá, que continuará como Director del IMO durante 10 años más, hasta su jubilación en 1981.

\section{Organización y funcionamiento}

La organización inicial de la Escuela, que no será la definitiva, queda establecida en el documento Bases Oficiales para la organización de la Escuela Especial de Orientación y 
Aprovechamiento del Excmo. Ayuntamiento de Valencia (Memoria, 1940, pp. 37-39), su primer reglamento orgánico, con solo 7 bases, el cual estará vigente hasta el curso 1943-44. En junio de 1944, dicho documento orgánico sufre un profundo cambio, pasando de 7 a 25 bases o artículos (Bases, 1944), reglamento que perdurará hasta la transformación de la EEOA en IMO en 1971.

En las Bases de 1940 solo se establece que la Escuela tendrá un Director y 4 Profesores. Vicente García Llácer fue nombrado Director, y éste a su vez nombró Maestro y Maestra especializados en superdotados a Vicente García Pueyo y a Dolores Peñuelas Heras; Maestro y Maestra especializados en anormales a José Zaragozá Antich y a Desamparados López Vila (Memoria, 1940). Con este personal se inaugura la Escuela el 1 de octubre de 1940 empezando a funcionar organizada en dos secciones (A y B) y cuatro aulas de clase para la acogida, durante un curso académico, de niños y niñas previamente seleccionados de la población escolar de Valencia con escasos recursos económicos, sin que por ello perdieran sus derechos como alumnos de sus centros de procedencia. La sección A acogía a los niños y niñas con bajas dotaciones intelectuales y se separaban por sexos en dos clases con un máximo de 15 alumnos cada una. La sección B acogía a niños y niñas superdotados intelectualmente y se procedía del mismo modo, pero en este caso las clases eran de 30 alumnos como máximo. En total, y durante su funcionamiento, se escolarizaron un máximo de 90 alumnos por curso. Durante el año de permanencia en el Centro, los alumnos recibían una educación diferencial adaptada a sus necesidades, eran observados por sus maestros y se sometían al pase de baterías psicotécnicas en el Laboratorio. Según los resultados obtenidos, al finalizar el curso, la Escuela proponía a la «Comisión de Colaboración y Ayuda» a los alumnos mas idóneos para ser becados por el Ayuntamiento. Una vez aprobadas las propuestas, los niños seleccionados recibían una beca para continuar estudios en el centro más adecuado según sus capacidades y el informe orientador emitido por la Escuela. Las becas eran de 500 ptas./año para los alumnos superdotados que continuaban estudios en las enseñanzas medias, de 1.000 ptas./año para estudios universitarios y de 750 ptas./año para los de enseñanzas especiales. Durante los 31 años de funcionamiento de la Escuela hubo un total de 390 becarios, lo que supone una media aproximada de 12 becarios/año (Giner, 1991).

El organigrama definitivo de la Escuela viene determinado por la remodelación de 1944 de las Bases fundacionales, donde en la nueva Base 2 se dice: «De momento, el personal indispensable para este Centro es el siguiente:

a) Director.

b) Maestro técnico auxiliar de Laboratorio.

c) Maestro técnico auxiliar de Estadística.

d) Auxiliar de Laboratorio y Estadística.

e) Maestro y Maestra especializados en Superdotados.

f) Maestro y Maestra especializados en Anormales.

g) Sacerdote-consiliario, Asesor de estudios de los becarios.

h) Mecanógrafa-bibliotecaria.

i) Maestro artesano de taller y

j) Celadora» (Bases, 1944, pp. 11-12). 
Además de estos funcionarios a tiempo completo, la Escuela disponía de unos profesores especiales, a tiempo parcial, para atender a los alumnos en materias y actividades como: Francés, Dibujo, Música, Rítmica, Labores y Trabajos Manuales. Para completar este organigrama se contrataron nuevos especialistas, permaneciendo en sus cargos el personal contratado en 1940, excepto José Zaragozá que pasará a Maestro técnico auxiliar de Laboratorio y su vacante como Maestro especializado en anormales será ocupada por Salvador Pizcueta Renau; Vicente García Pueyo pasará a Maestro técnico auxiliar de Estadística y la vacante de Maestro especializado en superdotados será ocupada por José Palanca Zaragozá. Para los demás puestos se nombraron a los siguientes profesionales: Auxiliar de Laboratorio y Estadística, Luisa Irurzum Fernández; Mecanógrafa-bibliotecaria, Concepción Vivó Ivars; Sacerdote-consiliario, Asesor de estudios de los becarios, Juan Muñoz Cardona; Maestro artesano jefe de taller, Esteban Osset y Celadora, Angeles Ferrer Agüero. El Profesorado especial a tiempo parcial se cubrió con personal de plantilla de las escuelas regentadas por el Ayuntamiento del modo siguiente: Música, Vicente Adrián Brotons; Francés, Leopoldo Santamarta; Labores, Desamparados Monsolíu; Trabajos Manuales, Milagros Agrait; Dibujo, José Hernández Doce y Rítmica, Josefina Gil Solís (Bases, 1944, p. 21).

En la Base 3 se enumeran las distintas dependencias que funcionarán en la Escuela, que además de las aulas de clase serán las siguientes: «El Laboratorio Psicotécnico, el Gabinete de Estadística, la biblioteca infantil y de consulta, y los talleres. Centro vital de la Escuela será la Capilla» (Bases, 1944, p. 12).

El Laboratorio Psicotécnico fue regentado desde 1940 hasta junio de 1944 por Vicente García Llácer en colaboración con José Zaragozá. El Laboratorio fue diseñado y montado conjuntamente por ambos y dotado de pruebas y tests para la selección y orientación de los escolares, material psicotécnico procedente de la biblioteca personal de José Zaragozá (discípulo y colaborador de Emilio Mira y López desde 1936 hasta 1938), y de los contactos personales que durante el verano de 1940 Zaragozá mantuvo con: el Instituto Psicotécnico de la Excma. Diputación de Barcelona, el Instituto Médico Pedagógico del Dr. Córdoba (Barcelona), el Instituto de Anormales Mentales del Ayuntamiento de Barcelona y con el Gabinete Paidométrico del Colegio «San Ignacio» de Sarriá (Barcelona) dirigido por el P. Palmés. Los cuestionarios, pruebas y tests, que tras estos contactos y consultas se estimaron necesarias para construir las baterías de selección, la elaboración de psicogramas y la posterior orientación de los escolares fueron las siguientes: Cuestionario R. Marston, Cuestionario íntimo, Cuestionario Otis pequeño, Cuestionario Thurston-Mira, Hoja psicométrica A, Tests de Terman, Prueba del trazado, Tests de visualización de Yerkes, Prueba psicométrica Modelo B-2, Prueba Donnaievsky, Prueba llaves B-2, Prueba baldosas B-2, Pruebas cuadros B-2, Prueba de las PP, Relaciones gráficas de Decroly, Relaciones gráficas de MiraOrtega, Prueba de las substituciones, Tests de Porteus, Prueba de Witmer, Prueba Foamboard, Test de Moede y algunas otras pruebas de tipo escolar. Además se adquirió un material mínimo para montar el Laboratorio psicotécnico y hacerlo operativo, como: báscula de precisión, dinamómetro, extensiómetro, espirómetro, ambidextrógrafo de Moede, cubos de Knox, etc. (Memoria, 1940; Zaragozá, 1996).

El trabajo del Laboratorio consistía en preparar las baterías de tests, realizar el pase de pruebas de selección por los distintos colegios de Valencia a fin de reclutar el alumnado superdotado e infradotado que nutriría la matrícula de la Escuela, adaptar y normalizar los tests a la población valenciana, construir pruebas objetivas para determinar el rendimiento académico de los alumnos, proponer las candidaturas de los becarios y formular el consejo 
orientador al finalizar la escolaridad. Tan ingente tarea determinó la necesidad de dotar al Laboratorio con el personal especializado anteriormente citado. El Gabinete de estadística era una dependencia aneja al Laboratorio y su principal misión consistía en la tabulación y el tratamiento estadístico de los datos obtenidos en el pase de las baterías psicotécnicas y las pruebas objetivas de rendimiento académico, la adaptación de tests y la construcción de baremos para la población escolar valenciana (Zaragozá, 1996).

Desde el primer momento, y pese a las penurias económicas de la época, se organizó una valiosa y nutrida biblioteca. Esta se componía de un reducido y selecto fondo de obras pedagógicas y psicopedagógicas destinadas al profesorado, y de una sección de obras culturales y recreativas para uso del alumnado. En 1943 se recibían 25 revistas y el fichero bibliográfico contenía unas 5.000 fichas (Anónimo, 1943).

Vicente García Llácer cesa en su cargo por exigencias laborales en 1948, pues al ser también Director de la «Escuela Aneja» a la Escuela del Magisterio de Valencia, y Profesor de la asignatura de «Prácticas de Enseñanza» y aumentar considerablemente la matrícula de los alumnos de Magisterio a partir de 1947, tiene que dedicarse en exclusiva a estas actividades de formación de los futuros maestros. Al finalizar el curso 1947-48 también cesa en su cargo de Maestro especializado en superdotados José Palanca, que pasará a ser Maestro de la Escuela Aneja del Magisterio. García Llácer, fundador y primer director de la EEOA, la dotó de unas Bases programáticas, organizativas y funcionales, de un Plan pedagógico, de un programa psicotécnico y de unos métodos, técnicas e instrumentos de trabajo que la convirtieron en institución educativa modélica de España durante las décadas de los años 40 y 50 del s. XX.

El 2 Período se inicia en el curso 1948-49 con los nombramiento de José Zaragozá Antich como nuevo Director, en sustitución de García Llácer; María Olmos, como Maestra técnica auxiliar de Laboratorio; Jesús Ayuda Morales, como Maestro técnico auxiliar de Estadística y de Felipe Gaspar Olmos que ocupó la vacante de Maestro especialista en superdotados dejada por José Palanca. En este Período la Escuela va a alcanzar su punto culminante durante el curso 1955-56. En septiembre de dicho curso se inaugura y bendice la nueva capilla del centro por el Rdo. Jacinto Argaya, Obispo auxiliar de la Archidiócesis de Valencia, y en octubre, la Escuela recibió la visita del Ministro de Educación Nacional Sr. Ruiz Giménez.

El $3^{\circ}$ Período empieza tras la marcha de su director José Zaragozá a Hispanoamérica comisionado por la UNESCO al finalizar el curso académico 1956-57. El curso escolar 195758 comenzó con un nuevo director, D. Felipe Gaspar Olmos, que sustituyó a José Zaragoza y el organigrama de la Escuela en esta nueva etapa de su andadura quedó integrado por el siguiente profesorado: Maestra técnica auxiliar de Laboratorio: Desamparados Rius Bueno, en sustitución de María Olmos que cesó en el cargo; Maestra auxiliar de Estadística, Pilar Escribano Lázaro, en sustitución de Jesús Ayuda Morales, que en 1953 había marchado a Barcelona para dirigir el Laboratorio de Psicología escolar del recién creado Instituto Municipal de Educación de Barcelona; Auxiliar de Laboratorio y Estadística, vacante; Maestro especializado en superdotados, Felipe Gaspar Olmos; Maestra especializada en superdotados, Dolores Peñuelas Heras; Maestro especializado en anormales, Cecilio Zurriaga Mira, en sustitución de Salvador Pizcueta; Mecanógrafa-bibliotecaria: vacante. Sacerdote-consiliario, Moisés Sánchez García en sustitución de Juan Muñoz. Maestro artesano jefe de taller, Esteban Osset y Celadora, Ángeles Ferrer Agüero. El Profesorado especial a tiempo 
parcial se cubrió con personal de plantilla de las escuelas regentadas por el Ayuntamiento del modo siguiente: Música, Vicente Adrián Brotóns; Francés: Regina Alcover Sebastián en sustitución de Leopoldo Santamarta; Labores, Cecilia Martí Benito en sustitución de Desamparados Monsolíu; Dibujo, Francisco Calatayud Llobell que en el curso 1945-46 sustituyó a José Hernández,; Trabajos manuales, Rítmica y Taquigrafía quedaron vacantes (Zaragozá, 1996).

Este Período se caracteriza por el estancamiento de la EEOA y su posterior decadencia, cerrando las aulas de la sección A (alumnos con n.e.e.) al finalizar el año académico 196061. Crisis de la que ya no se recuperará, a pesar de que a su regreso de América en 1968 José Zaragozá volverá a asumir la dirección de la Escuela, que desaparece definitivamente al finalizar el curso 1970-71, al ponerse en marcha la nueva ordenación del sistema educativo español con la implantación de la EGB y la escolarización obligatoria hasta los 14 años, de acuerdo con lo establecido en la recién aprobada Ley General de Educación (1970) y convertirse la EEOA en Instituto Municipal de Orientación (IMO) por decisión de la Junta Municipal del Ayuntamiento de Valencia en 1971 (Giner, 1991).

\section{Actividades}

En este apartado analizaremos exclusivamente la labor y actividades de Orientación psicopedagógica desarrolladas por la Escuela desde su fundación hasta su reconversión en Instituto Municipal de Orientación en 1971. El programa pedagógico que siguió la Escuela, de acuerdo con el ideario y los principios doctrinales contenidos en sus Bases (1944), se halla minuciosa y ampliamente expuesto en la Tesis doctoral que bajo mi dirección realizó $\mathrm{M}^{\mathrm{a}}$ José Giner (1991 y 1993) y a la cual remito al lector interesado en conocer sus actividades de enseñanza-aprendizaje.

Durante el $1^{\circ}$ Período (1940-48), bajo la dirección de García Llácer, la actividad de la Escuela se centra exclusivamente en la selección, clasificación, escolarización (durante un curso académico), orientación escolar y asignación de becas a los alumnos más destacados.

El programa psicotécnico de la Escuela estaba perfectamente diseñado (baterías de tests), y los resultados se recogían en una ficha acumulativa, parte de un amplio expediente escolar que incluía además, las notas académicas (pruebas objetivas) e información observacional sobre la conducta de los escolares a cargo de sus profesores. El Plan pedagógico de la Escuela se basaba en los conocimientos científicos de la psicología evolutiva y diferencial, estaba programado para atender las necesidades de los niños y niñas (infradotados, clases A y superdotados, clases B) entre los siete y once años, y se adecuaba a los principios ideológicos (nacional-catolicismo) y a las corrientes pedagógicas (herbartiana) del momento.

Durante el $2^{\circ}$ Período, las actividades de la Escuela no sufren variación alguna y se siguen las directrices marcadas en sus Bases (1944), se mantiene el mismo programa pedagógico y psicotécnico con su doble compromiso: «desarrollo integral de las aptitudes y facultades de sus alumnos y emisión del Consejo orientador al finalizar el curso». El primer objetivo se cumplía con el desarrollo del programa pedagógico y el segundo por medio de un minucioso y complejo programa psicotécnico que se sintetizaba en un completo psicobiograma, primera base de datos para la elaboración del Consejo orientador. Sin embargo, la Escuela no consideraba suficiente esta información técnica, que se completaba con el cono- 
cimiento directo del niño a través de la observación directa y sistemática de su escolaridad que se concretaba en el informe aportado por sus maestros.

El programa psicotécnico se realizaba a lo largo de todo el curso durante la jornada escolar de los sábados. Las pruebas colectivas se realizaban en el aula, mientras que las individuales y de aparatos se aplicaban en el Laboratorio, al que acudían los alumnos que iban a ser explorados, mientras el resto permanecía en clase. Los responsables del programa eran los Maestros/as técnicos auxiliares de Laboratorio y Estadística, que además de aplicar y corregir las pruebas, tabular, comprobar e interpretar los resultados, se encargaban de controlar los procesos de enseñanza-aprendizaje y de investigar las características ambientales y familiares de los escolares (Zaragozá, 1996).

Además del cumplimiento de todas estas actividades, José Zaragozá da una nueva dimensión a la labor de la EEOA, propiciando su proyección social y científica, tanto a nivel municipal como nacional e incluso internacional, por medio de:

a) Revisión de baterías y adaptación de tests. Desde el momento en que José Zaragozá se hizo cargo de la dirección de la Escuela y partiendo del banco de datos del Laboratorio psicotécnico, se inician una serie de trabajos para la revisión, actualización, adaptación y normalización de los test que se utilizaban para la selección y orientación de su alumnado, en colaboración con los estudiantes de prácticas de la Escuela Normal del Magisterio de Valencia, así como otros trabajos sobre la dotación intelectual de la población escolar valenciana (Zaragozá, 1948, 1949, 1950, 1950a y 1951). Para la medida de las aptitudes de los alumnos de la sección A (alumnos con n.e.e.), se utilizaba el Perfil Psicográfico de Vermeylen. La batería psicotécnica de aptitudes a la que se sometían los alumnos de la sección B (superdotados) era la siguiente: Atención: Reactivo de Toulouse-Pieron, Prueba de Kräpelin y Prueba de Rupp. Dibujo: Prueba de Wartergg y Prueba de Westag Habilidad manual: Cilindro de Witmer, Ambidextrógrafo de Moede, Ambidextrógrafo de Rupp y Prueba del trazado de Borrás-Granada. Inteligencia abstracta: Cuestionario multifactorial Thurston-Mira, Cuestionario Otis (pequeño) y Test de Decroly. Inteligencia de cálculo: Cuestionario multifactorial Thurston-Mira, Cuestionario Otis (pequeño) y Prueba analítico abstracta de Meili. Inteligencia espacial-gráfica: Prueba de las llaves, Prueba de las baldosas y Prueba de los cuadrados. Inteligencia espacio-manual: Formboard, Puzzle de madera, Cubos de Knox y Cilindro de Witmer. Inteligencia práctica: Tableros de órdenes. Inteligencia técnica: Series de Donnaiewsky y Caja de Decroly. Inteligencia verbal: Prueba de las PP, Cuestionario multifactorial Thurston-Mira y Cuestionario Otis (pequeño). Memoria: Prueba de memoria visual y Prueba de memoria auditiva. Visualización: Cubos de Yerkes (Giner, 1991 y 1993).

b) Programas de información escolar y ocupacional. Estos programas, iniciados en 1952, se organizaron en colaboración con la Inspección de Enseñanza Primaria, tanto a nivel municipal como nacional, realizando una amplia labor de información escolar y vocacional dirigida a los alumnos del último año de escolaridad obligatoria. Dentro de un plan general de actuación, se daban charlas y conferencias sobre las oportunidades de proseguir estudios en los centros de enseñanza media y de formación profesional y a todos los asistentes se les entregaba un completo folleto informativo (Ríus, Peñuelas y Zaragozá, 1952). A su vez, se requería a los alumnos a 
contestar unos cuestionarios sobre la información recibida. En la Escuela también se realizaban sesiones informativas a cargo de profesionales para los becarios del Centro y los alumnos de los colegios que lo solicitaban. En estos coloquios, los profesionales invitados, con experiencia probada en el ejercicio de carreras de grado medio y superior desarrollaban «autobiografías vocacionales», con todo lo positivo que tiene el relato directo y vivo de experiencias personales (Zaragozá, 1978).

c) Organización de cursos de Psicotecnia para maestros y profesores. En el curso académico 1952-53 se organizó el I Curso de «Introducción a la Psicotecnia escolar». Fue patrocinado por la Dirección General de Enseñanza Primaria y colaboraron los Institutos «San José de Calasanz» de Pedagogía, con Víctor García Hoz y el Instituto Nacional de Psicotecnia, con José Germain y José Mallart y otros destacados profesionales de la Pedagogía y la Psicología. Estos cursos se convocarían anualmente hasta el año académico 1956-57 en que José Zaragozá marchó a Hispanoamérica. Durante sus cuatro ediciones participaron como profesores de los mismos, además de García Hoz, Germain y Mallart, prestigiosos especialistas, como: Jesusa Pertejo, Ricardo Marín, José Todolí, Julián Sánchez Jiménez, Francisco Domingo, José Zaragozá, etc. El contenido de los cursos presentaba un atractivo temario que comprendía el estudio de la personalidad del niño y del adolescente, la Orientación personal, escolar y profesional, técnicas psicoanalíticas, los registros escolares y la medida de las aptitudes. Estas enseñanzas teóricas se completaban con prácticas de laboratorio para el estudio y la aplicación de tests. Los cursos despertaron un gran interés entre los docentes de la ciudad de Valencia, como lo demuestra el número de matriculados en los mismos (Zaragozá, 1983):

\begin{tabular}{|c|c|}
\hline Curso académico & $\mathbf{N}^{\mathbf{0}}$ de matriculados \\
\hline $1952-53$ & 150 \\
\hline $1953-54$ & 166 \\
\hline $1954-55$ & 187 \\
\hline $1955-56$ & 180 \\
\hline
\end{tabular}

Con la marcha de Zaragozá a Hispanoamérica se interrumpieron definitivamente estas actividades formativas que desgraciadamente ya no volverían a reanudarse.

d) Escuela de Padres. En el curso 1954-55 para mejorar la atención a los escolares del Centro se creó la «Escuela de Padres» como resultado de las reuniones informativas que periódicamente mantenía la dirección y el claustro de profesores de la EEOA con los padres de sus alumnos. Surge como respuesta a las inquietudes y necesidades manifestadas en dichas reuniones ante el problema de la educación de los hijos, teniendo en cuenta las características especiales de los alumnos de la Escuela. Diversos profesionales y especialista intervinieron en las sesiones organizadas en torno a la educación familiar, se impartieron cursillos monográficos sobre problemáticas específicas de las relaciones familiares. Los cursillos y conferencias sobre el tema se recopilaron y dieron lugar a dos publicaciones: La orientación profesional en la familia (VV.AA., 1956) que recogió las intervenciones de J. Alcamí, R. Marín, V. 
García Hoz, J. Pertejo, J. Ruiz, C. Salazar y J. San Valero y Familia, niño y escuela (Sánchez Jiménez, 1956) fruto de un cursillo del mismo título. Con el cambio de dirección en 1957, la «Escuela de Padres» siguió funcionando, pero ya no se organizaron cursillos formativos y se volvió a la antigua dinámica de las reuniones periódicas con los profesores del centro para tratar problemas relativos a la escolaridad de los alumnos (Zaragozá, 1983).

e) Publicaciones. En 1950 aparece la Revista de Psicología y Pedagogía Aplicadas, órgano y portavoz oficial de la Escuela, con periodicidad semestral y con la finalidad de «llevar al campo de la educación los avances y aplicaciones prácticas de la Psicología y la Pedagogía». Su fundador y director único fue José Zaragozá. La revista dejaría temporalmente de publicarse en 1957, cuando aquel marchó a Hispanoamérica comisionado por la UNESCO. Durante estos siete años, con una periodicidad semestral y la colaboración de especialistas nacionales y extranjeros, se publicaron 14 números con un total de 39 artículos cuya relación completa se presenta en el Anexo. Sus contenidos se estructuraron en las siguientes secciones: Editorial, Artículos, Trabajos de laboratorio, Informaciones comentadas, Resumen de actividades y su proyección, Documentación, Fichas bibliográficas, Cursos, conferencias y congresos, y Notas y noticias. La revista se complementaba con un folleto titulado Documentación Psicopedagógica que recogía recensiones de libros y artículos especializados. La revista volvió a reeditarse cuando Zaragozá regresa a España, y el n ${ }^{\circ} 15$ no aparecerá hasta 1975 como portavoz del Instituto Municipal de Orientación (IMO). Durante este primer período (1950-56) se estableció un ambicioso programa de intercambios. La Revista de Psicología y Pedagogía Aplicadas llegó a intercambiarse con 196 publicaciones periódicas diferentes, tanto nacionales como extranjeras, que vinieron a enriquecer los fondos especializados de la Biblioteca de la Escuela. Un detallado estudio bibliométrico de la revista fue realizado por $\mathrm{M}^{\mathrm{a}}$ José Giner (1991 y 1993) como parte de su tesis doctoral. También se publicaba un folleto periódico titulado Orientación, órgano de los becarios de la Escuela y la «Colección Psicopedagógica», que editó en total cinco monografías: Gnosograma (Zaragozá, 1954), Problemas y perspectivas de la Orientación Profesional (Germain, 1956), La Orientación profesional en la familia (VV.AA., 1955), Familia, niño y escuela (Sánchez Jiménez, 1956) y Psicograma (Zaragozá, 1957).

La EEOA vive durante estos años sus mejores momentos en una clara línea ascendente. Su bien ganado prestigio trasciende mas allá de los límites municipales y se extiende a nivel nacional e incluso internacional, difundido por la Revista de Psicología y Pedagogía Aplicadas que desde su aparición inició una decidida campaña de difusión de sus cursos, experiencias, trabajos e investigaciones, así como de intercambio con otras publicaciones científicas especializadas en Psicopedagogía.

A partir del curso 1957-58 el nuevo equipo docente, bajo la dirección de Felipe Gaspar, siguió trabajando en la selección, orientación y educación de los niños y niñas de las secciones A y B, pero dejó de organizar toda una serie de actividades complementarias (cursos de formación, escuela de padres, cursillos psicotécnicos, etc) y también dejó de publicar la Revista de Psicología y Pedagogía Aplicadas y la colección de monografías sobre temas de orientación psicopedagógica. A su regreso de Hispanoamérica en 1968, José Zaragozá volvió a retomar la dirección de la Escuela durante los cursos 1968-69, 1969-70 y 70-71, pero ya no se volvieron a recuperar las actividades complementarias desarrolladas anteriormente, 
excepto la publicación de la Revista, debido a los recortes presupuestarios a que fue sometida la Escuela por el Ayuntamiento de Valencia, patrocinador y mecenas de la misma. En 1971, la EEOA cerraría definitivamente sus puertas y se convertiría en Instituto Municipal de Orientación (IMO), cuya creación, organización, funcionamiento y actividades analizaremos a continuación.

\section{EI Instituto Municipal de Orientación (IMO) del Excmo. Ayuntamiento de Valencia (1971-1981)}

\section{Orígenes}

A partir del curso 1970-71, con la implantación de la reforma propuesta por la Ley General de Educación (1970) la Escuela Especial de Orientación y Aprovechamiento (EEOA) se transforma en Instituto Municipal de Orientación (IMO), por lo que sus fines tuvieron que adaptarse a las nuevas necesidades y quedaron reducidos básicamente a tres:

a) Orientar escolar y profesionalmente al alumnado de las escuelas públicas de EGB de la ciudad de Valencia que lo solicitasen, ayudando al profesorado a completar los datos psicológicos del Extracto del Registro Personal del Alumno (ERPA).

b) Investigar en el campo de la psicopedagogía infantil y juvenil, especialmente en lo referente a la problemática del escolar valenciano.

c) Mejorar la biblioteca, promover un centro de Documentación e Información Psicopedagógica, reeditar la Revista de Psicología y Pedagogía Aplicadas, que nacida en 1950 dejó de editarse en 1957, y restablecer los intercambios con otros centros y revistas científicas.

Ya en 1969, como consecuencia de la publicación del «Libro Blanco» (MEC, 1969), en el que se analizaba críticamente la situación educativa y se esbozaba el inminente cambio del sistema educativo en España, la EEOA tuvo que replantearse sus objetivos, estructura y actividades de enseñanza primaria con los alumnos superdotados intelectualmente que seleccionaba de las escuelas de Valencia, y que becados por el Ayuntamiento, preparaba anualmente en sus dos aulas (máximo de 30 alumnos por aula) para su posterior acceso a la enseñanza media. Así pues, en 1969 se iniciaría la preparación y redacción de un proyecto para transformar la EEOA en Instituto Municipal de Orientación (IMO), a la vez que se replanteó su colaboración con los profesores y los programas de prácticas de los alumnos de la especialidad de Orientación escolar de la Sección de Pedagogía de la Facultad de Filosofía y Letras de la Universidad de Valencia.

\section{Principios doctrinales}

En cuanto a sus principios doctrinales y metodología, el IMO no sufrió cambios sustanciales con respecto a los que rigieron las actividades de la EEOA, ya que su director, José Zaragozá, a su regreso de Hispanoamérica a finales de 1968, retomó las actividades que había dejado aparcadas al marcharse y continuó en la misma línea de actuación sin introducir innovaciones significativas, siguiendo en la práctica la corriente «psicotécnica europea del sujeto y del objeto» y la medida de rasgos y factores heredada del Institut de Barcelona, 
donde fue discípulo directo de Mira y López durante los años críticos de nuestra Guerra Civil (1936-39) (Benavent, 1996 y 2000), aunque en esta nueva etapa se pretende ... «un resultado beneficioso para el escolar y tener información objetiva sobre las posibilidades del individuo, problemas que puede presentar en su desarrollo psicobiológico, carácter e intereses, tratando de conseguir la aplicación consciente, en el adolescente, de una orientación no directiva y autorresponsable. El individuo debe ser él quien haga síntesis, tome decisiones y se comprometa, de acuerdo con los hechos que determinan sus elecciones» (Zaragozá, 1975a, p. 6).

A partir de este momento, el IMO, con escaso personal y recursos, se limitó a atender las demandas de orientación escolar y profesional de las escuelas de la ciudad de Valencia y de algunas poblaciones de la provincia que voluntariamente solicitaban este tipo de servicios. Para ello se utilizaban las mismas baterías y recursos psicotécnicos de la antigua EEOA, normalizando pruebas, revisando y actualizando baremos, tratando estadísticamente los datos de sus archivos para elaborar trabajos que se publicaban en la Revista de Psicología y Pedagogía Aplicadas.

\section{Organización}

La EEOA, que ya había abandonado la atención a los niños de educación especial, en los últimos años de su existencia (1968-1971), estuvo dedicada casi exclusivamente a las actividades de selección y orientación de los alumnos superdotados de las escuelas públicas de la ciudad de Valencia. Al perder sus competencias docentes en la EGB, como consecuencia de la implantación de la reforma educativa de la LGE (1970), sufrió un drástico recorte de plantilla y empezó a denominarse oficiosamente Instituto Municipal de Orientación (IMO) en 1971. En 1975 el IMO se traslada, desde la vieja casona de la calle Salvador Giner $n^{a} 5$ donde estuvo ubicada la EEOA desde 1947 (edificio habilitado que disponía de amplio espacio para aulas y laboratorios), a la sexta planta del edificio $\mathrm{n}^{\mathrm{o}} 14$ de la misma calle donde permaneció hasta 1981, año en que se transformó y pasó a denominarse Instituto Municipal de Educación (IME). Como consecuencia de estos cambios se suprimieron las Enseñanzas Especiales (Francés, Música, Trabajos Manuales y Taller); se cancelaron los contratos del profesorado que impartía clases de educación primaria y el personal de plantilla quedó limitado al Director, José Zaragozá, a la Maestra especialista en estadística, Pilar Escribano, a la Auxiliar de estadística, Amelia Olagüe, y a los Maestros, Dolores Peñuelas Heras, Desamparados Rius Bueno y Felipe Gaspar Olmos, los cuales permanecieron en activo hasta su jubilación en 1980, 1983 y 1985 respectivamente. Después de un corto período de transición y adaptación al nuevo marco legal establecido por la LGE de 1970, la denominación de Instituto Municipal de Orientación fue reconocida oficialmente en 1976, siendo Concejala de Educación del Excmo. Ayuntamiento de Valencia Leonor Vilar, al aprobarse por el Consejo Municipal el proyecto que había presentado en 1974 su Director José Zaragozá (Escribano, 2001).

\section{Actividades}

La actividad del IMO durante esta etapa (1971-1981) se centró casi exclusivamente en las escuelas de EGB de la ciudad de Valencia y de algunas localidades de la provincia que 
solicitaban sus servicios, aplicando una batería de tests que permitía cumplimentar los datos psicotécnicos del Extracto del Registro Personal del Alumno (ERPA), analizar sus intereses vocacionales y profesionales, y realizar una labor de seguimiento que permitía formular un consejo orientador al final de la escolaridad obligatoria. En la tabla adjunta (Fig. 1) se presenta un resumen estadístico de la labor psicotécnica realizada con la colaboración de los alumnos en prácticas de la especialidad de «Orientación Escolar» de la Sección de Pedagogía de la Facultad de Filosofía y Ciencias de la Educación y de la Escuela Universitaria del Profesorado de EGB de la Universidad de Valencia, previamente preparados por medio de un cursillo sobre «Evaluación estadística y prácticas psicopedagógicas» que con una duración de 15 días se impartía en el IMO a grupos reducidos (máximo 30 alumnos) por José Zaragozá, Pilar Escribano, Felipe Gaspar y Amelia Olagüe. Esta tarea se complementaba con el estudio de casos, en especial, con alumnos de los primeros cursos de la EGB (Anónimo, 1975).

\begin{tabular}{|c|c|c|}
\hline Curso & $\mathbf{N}^{\mathbf{0}}$ de alumnos de EGB examinados & $\mathbf{N}^{\mathbf{0}}$ de pruebas aplicadas \\
\hline $1972-73$ & 2.469 & 13.752 \\
\hline $1973-74$ & 2.636 & 13.192 \\
\hline $1974-75$ & 3.601 & 19.178 \\
\hline $1975-76$ & 3.350 & 19.822 \\
\hline $1976-77$ & 4.664 & 22.792 \\
\hline $1977-78$ & 4.994 & 21.474 \\
\hline $1978-79$ & 3.861 & 20.395 \\
\hline $1979-80$ & 3.645 & 15.024 \\
\hline $1980-81$ & 3.407 & 21.683 \\
\hline
\end{tabular}

Tabla: Elaboración propia.

Fuente: Revista de Psicología y Pedagogía Aplicadas (1975-1981).

FIGURA 1.

Tabla resumen de los alumnos de EGB examinados y del número de pruebas aplicadas durante el período de 1972 a 1981.

El IMO también se ocupó de impartir cursillos especializados sobre autobiografías profesionales (Anónimo, 1975a) y sobre documentación e investigación psicopedagógica, especialmente en el campo de la enseñanza, y colaboró estrechamente con el ICE de la Universidad de Valencia, desde el curso 1972-73, en la organización de actividades psicopedagógicas, seminarios y cursos especializados sobre técnicas de medida, trabajo intelectual, psicomotricidad, etc., para la formación y perfeccionamiento del profesorado y los orientadores escolares (Orts, 1980). Para llevar a cabo todas estas actividades se contaba, además de la reducida plantilla de personal del IMO, con el ya mencionado apoyo desinteresado de los alumnos en prácticas de la Escuela Universitaria del Profesorado de EGB y de los de «Orientación Escolar», de la Sección de Pedagogía de la Facultad de Filosofía y Ciencias de la Educación, y de algunos profesores de Psicología y Pedagogía de la Universidad de Valencia. 
Gracias a estas colaboraciones y a la financiación del Ayuntamiento de Valencia se dio continuidad y contenido a la Revista de Psicología y Pedagogía Aplicadas, que no se editaba desde 1956, y que apareció de nuevo en 1975. En esta segunda etapa editorial (19751981), «... la Revista de Psicología y Pedagogía Aplicadas, proseguirá aportando las realizaciones del Instituto Municipal de Orientación en el estudio científico de las técnicas para el mejor conocimiento de la personalidad del escolar. Los trabajos de psicopedagogía, estudio y adaptación de tests, escalas e información escolar y profesional que se preparen y toda temática útil a la docencia irán apareciendo en sus páginas» (Zaragozá, 1975, p. 6). La Revista, como órgano oficial y portavoz del IMO, siguió bajo la dirección de José Zaragozá hasta su jubilación, conservando la misma finalidad, formato y estructura de su primera etapa (1950-1956) hasta el no 26 (1981). Durante este período, la Revista de Psicología y Pedagogía Aplicadas publicó 12 números con un total de 38 artículos de variada temática psicopedagógica, y 19 trabajos empíricos y/o experimentales de laboratorio sobre la problemática psicopedagógica del escolar y la construcción, adaptación, normalización y validación de tests, cuya relación completa se presenta en el Anexo. En 1982 la Revistas no se editó, pero a partir del $n^{\circ} 27$ (1983), se inaugura una nueva etapa y siguió publicándose con la misma cabecera, pero como portavoz oficial del Instituto Municipal de Educación (IME) bajo la dirección de José Luis Domenech, cambiando sus objetivos, secciones, formato y estructura interna, y perdiendo definitivamente su carácter de publicación especializada en Orientación psicopedagógica para desaparecer en 1987.

Simultáneamente se fue ampliando el Centro de Documentación e Información, sección del IMO, que divulgaba sus actividades por medio de Documentación Psicopedagógi$c a$, publicación periódica que aparecía como volumen suplementario de los números de la Revista de Psicología y Pedagogía Aplicadas hasta el no 19-20 del año 1978 en que Documentación Psicopedagógica se incorpora e integra en dicho número como una nueva sección del mismo, situación que se prolongará hasta la desaparición de la revista en 1987. En ella aparecían recensiones de libros y artículos seleccionados de las revistas obtenidas en los intercambios, gracias a la colaboración de los alumnos en prácticas de la Universidad de Valencia que se encargaban de hacer los resúmenes. En todo este proceso documental se seguía la clasificación bibliográfica decimal de la Oficina Internacional de Educación de Ginebra, lo que facilitaba el canje de información científica con otras instituciones y organismos nacionales e internacionales dedicados a la Orientación psicopedagógica. Estos intercambios documentales y el haber trabajado José Zaragozá como Experto de la UNESCO en Hispanoamérica, facilitaron la firma en 1977 de un convenio de colaboración entre el IMO y el Departamento de Educación de la Organización de Estados Americanos (OEA), a través del Instituto de Cooperación Iberoamericano, para la formación de especialistas becados por la OEA en el IMO con el objeto de seguir programas de formación y perfeccionamiento en Orientación psicopedagógica y realizar trabajos de investigación (Zaragozá, 1978).

Al carecer el IMO del necesario apoyo económico para renovar y actualizar los materiales e instrumentos y ampliar la plantilla de personal especializado, junto a los cambios de ideología política que informaron la educación en la «España de la transición» y la coincidencia de la jubilación de su director José Zaragozá, determinaron su transformación en Instituto Municipal de Educación (IME) en 1981.

Dos años más tarde, el 20 mayo de 1983, en el Club Náutico de Valencia, tuvo lugar una Cena-Homenaje promovida por la Consellería de Cultura, Educación y Ciencia, en honor 
de José Zaragozá en justo reconocimiento de los servicios prestados durante su dilatada trayectoria profesional, pronunciando un emotivo discurso de despedida Pilar Escribano (Anónimo, 1983a).

\section{Referencias bibliográficas}

Anónimo (1943): «La Escuela de Orientación y Aprovechamiento, Valencia». Revista Nacional de Educación, 3 (31), pp. 97-103.

Anónimo (1950): «Propósitos». Revista de Psicología y Pedagogía Aplicadas, 1 (1), p. 5.

Anónimo (1975): «Programa de los cursos para el alumnado de Escuelas Universitarias del Profesorado». Revista de Psicología y Pedagogía Aplicadas, 8 (15), pp. 155-156.

Anónimo (1975a): «Curso de autobiografías profesionales». Revista de Psicología y Pedagogía Aplicadas, 8 (15), p. 157.

Anónimo (1983): «Editorial». Revista de Psicología y Pedagogía Aplicadas, 14 (27), pp. 5-7.

Anónimo (1983a): «Homenaje a D. José Zaragozá Antich». Revista de Psicología y Pedagogía Aplicadas, 14 (27), p. 118.

Bases. Escuela Especial de Orientación y Aprovechamiento (1944): Valencia: Excmo. Ayuntamiento de Valencia.

Benavent, J. A. (1996): La Orientación Psicopedagógica en España. Vol. I: Desde sus orígenes hasta 1939. Valencia: Edit. Promolibro.

Benavent, J. A. (2000): La Orientación Psicopedagógica en España. Vol. II: Desde 1939 hasta la Ley General de Educación de 1970. Valencia: Edit. Promolibro.

Claparède, E. (1924): La Orientación profesional. Sus problemas y sus métodos. Madrid: Edit. «La Lectura».

Claparède, E. (1927): Psicología del niño. Madrid: Edit. Beltrán.

Chleusebairgue, A. (1934): Orientación Profesional. 2 vol. Barcelona: Edit. Labor.

Escribano, P. (2001): Testimonio personal. Entrevistas mantenidas por los autores de este trabajo con Dña. Pilar Escribano Lázaro, Becaria de la Escuela Especial de Orientación y Aprovechamiento del Ayuntamiento de Valencia (EOAA), Maestra municipal, Maestra especializada en estadística de la EEOA, Licenciada en Pedagogía, personal de plantilla del IMO, del IME y responsable hasta su jubilación del Gabinete Psicopedagógico Municipal del Ayuntamiento de Valencia.

García Llácer, V. (1943): «Orientación profesional académica». Revista Nacional de Educación, 3 (32-33), pp. 79-100.

Germain, J. (1956): Problemas y perspectivas de la Orientación profesional. Valencia: Excmo. Ayuntamiento de Valencia. Escuela Especial de Orientación y Aprovechamiento.

Giner, Mª J. (1991): La Escuela Especial de Orientación y Aprovechamiento del Excmo. Ayuntamiento de Valencia: Una experiencia de Orientación Educativa. 2 vol. Valencia: Universitat de València. Facultad de Filosofía y Ciencias de la Educación. (Tesis doctoral inédita).

Giner, Mª. J. (1993): La Escuela Especial de Orientación y Aprovechamiento del Excmo. Ayuntamiento de Valencia: Una experiencia de Orientación Educativa. Valencia: Universitat de València. Departamento de Didáctica y Organización Escolar. (Tesis doctoral en microfichas).

Marañon, G. (1936): Vocación y ética. $2^{\mathrm{a}}$ ed. Madrid: Espasa-Calpe.

Memoria de la Escuela Especial de Orientación y Aprovechamiento (1940). Valencia: Excmo. Ayuntamiento de Valencia. 
Orts, J. M. (1980): «Actividades del I.C.E. de la Universidad de Valencia en colaboración con el Instituto Municipal de Orientación de Valencia». Revista de Psicología y Pedagogía Aplicadas, 12 (23-24), pp. 161-163.

Revista de Psicología y Pedagogía Aplicadas. Escuela Especial de Orientación y Aprovechamiento del Excmo. Ayuntamiento de Valencia. Valencia. 1 (1-2), 1959; 2 (3-4), 1951; 3 (5-6), 1952; 4 (78), 1953; 5 (9-10), 1954; 6 (11-12), 1955 y 7 (13-14), 1956. (La revista dejo de publicarse desde 1957 hasta 1974. Instituto Municipal de Orientación del Excmo. Ayuntamiento de Valencia. Valencia. 8 (15 y 16), 1975; 9 (17-18), 1976; (no se editó en 1977); 10 (19-20), 1978; 11 (21-22), 1979; 12 (23-24), 1980; y 13 (25-26), 1981.

Rius, A., Peñuelas, J. y Zaragozá, J. (1952): Orientación Escolar, 1952. Valencia: Excmo. Ayuntamiento de Valencia. Escuela Especial de Orientación y Aprovechamiento.

Sánchez Jiménez, J. (1955): Familia, niño, escuela. Valencia: Excmo. Ayuntamiento de Valencia. Escuela Especial de Orientación y Aprovechamiento.

Spranger, E. (1935): Psicología de la edad juvenil. $2^{\mathrm{a}}$ ed. Madrid: Ed. Revista de Occidente.

VV.AA. (1955): La Orientación profesional en la familia. Valencia: Excmo. Ayuntamiento de Valencia. Escuela Especial de Orientación y Aprovechamiento.

Zaragozá, J. (1948): «Las Series de Cinemática de Donnaiewsky». Revista de Psicología General y Aplicada, 3 (7), pp. 541-554.

Zaragozá, J. (1949) «Datos para el estudio de la aptitud y tendencia del escolar». Revista Española de Pedagogía, 8 (32), pp. 649-654.

Zaragozá, J. (1950): «Los índices de oligofrenia en Valencia. Su asistencia». Revista de Psicología y Pedagogía Aplicadas, 1 (1), pp. 49-63.

Zaragozá, J. (1950a): «Graduación de un test psicométrico». Revista de Psicología General y Aplicada, 5 (16), pp. 755-770.

Zaragozá, J. (1951): «El problema de los bien dotados y su atención en Valencia». Revista de Psicología y Pedagogía Aplicadas, 2 (3), pp. 49-63.

Zaragozá, J. (1952): «El censo escolar de Valencia». Revista de Psicología y Pedagogía Aplicadas, 3 (5), pp. 9-30.

Zaragozá, J. (1954): Gnosograma. Valencia: Excmo. Ayuntamiento de Valencia. Escuela Especial de Orientación y Aprovechamiento.

Zaragozá, J. (1957): Psicograma. Valencia: Excmo. ayuntamiento de Valencia. Escuela Especial de Orientación y Aprovechamiento.

Zaragozá, J. (1975): «Editorial». Revista de Psicología y Pedagogía Aplicadas, 8 (15), pp. 5-6.

Zaragozá, J. (1975a): «La Orientación en 1975: Función actual de la Orientación» (Editorial). Revista de Psicología y Pedagogía Aplicadas, 8 (16), pp. 5-7.

Zaragozá, J. (1978): «Instituto Municipal de Orientación». Revista de Psicología y Pedagogía Aplicadas, 10 (19-20), pp. 133-140.

Zaragozá, J. (1983): «El Instituto Municipal de Orientación de Valencia. Una nota histórica». Revista de Psicología General y Aplicada, 38 (180), pp. 215-226.

Zaragozá, J. (1996): Testimonio personal. Varias entrevistas mantenidas por el autor de este trabajo con D. José Zaragozá. 


\section{Legislación}

Ley 14/1979, de 4 de agosto: General de Educación y Financiamiento de la Reforma Educativa, (BOE del 6 de agosto).

\section{Anexo}

\section{Articulos publicados en la revista de Psicología y Pedagogía Aplicadas (Primera etapa: 1950-1956).}

\section{0}

Garmendía de Otaola, A.: «Clasificación de los talentos dentro del paradigma psicológico». Revista de Psicología y Pedagogía Aplicadas, 1 (2), pp. 29-39.

Mallart, J.: «Pre-orientación profesional e iniciación laboral sistemática». Revista de Psicología y Pedagogía Aplicadas, 1 (1), pp. 23-47.

Mallart, J.: «Aplicaciones de la Psicología: Orientación escolar, orientación profesional, selección escolar y formación profesional». Revista de Psicología y Pedagogía Aplicadas, 1 (2), pp. 41-60.

Del Olmo, F.: «Evaluación del trabajo escolar». Revista de Psicología y Pedagogía Aplicadas, 1(2), pp. 11-27.

Pieron, H.: «El problema de la inteligencia». Revista de Psicología y Pedagogía Aplicadas, 1 (1), pp. 9-22.

Zaragozá, J.: «Los índices de oligofrenia en Valencia: su asistencia». Revista de Psicología y Pedagogía Aplicadas, 1 (1), pp. 49-63.

\section{1}

Alcayde, F:. «Variantes fisiológicas en sujetos emocionados». Revista de psicología y Pedagogía Aplicadas, 2 (3), pp. 21-26.

Gómez-Ferrer, P.: «Valencia necesita un hospital-jardín para niños». Revista de Psicología y Pedagogía Aplicadas, 2 (4), pp. 243-261.

Lobet, E.: «Experiencias belgas en materia de Orientación escolar y profesional». Revista de Psicología y Pedagogía Aplicadas, 2 (3), pp. 9-20.

Meschieri, L.: «Reseña de alguno de los recientes métodos del estudio de la personalidad humana en experimentación». Revista de Psicología y Pedagogía Aplicadas, 2 (4), pp. 201-217.

Sánchez Jiménez, J.: «Ensayo de las técnicas de medida objetiva del trabajo escolar en el grupo de niños Nuestra Señora de Begoña de Altos Hornos». Revista de Psicología y Pedagogía Aplicadas, 2 (4), pp. 219-241.

Zaragozá, J.: «El problema de los bien dotados y su atención en Valencia». Revista de Psicología y Pedagogía Aplicadas, 2 (3), pp. 27-40.

\section{2}

Bertrán, J.: «Posibles aplicaciones prácticas del test La rapidez de escritura». Revista de Psicología y Pedagogía Aplicadas, 3 (5), pp. 73-87. 
Forteza, J.: «El dibujo como test imaginativo en la selección de aprendices». Revista de Psicología y Pedagogía Aplicadas, 3 (6), pp. 251-261.

Garmendía de Otaola, A.: «El test de Roschard y el examen de los talentos». Revista de Psicología y Pedagogía Aplicadas, 3 (6), pp. 237-250.

Mallart, J.: «Los municipios y la iniciación profesional de los jóvenes». Revista de Psicología y Pedagogía Aplicadas, 3(5), pp. 31-48.

Mansuet, F.: «De los cocientes de inteligencia a la obligación escolar». Revista de Psicología y Pedagogía Aplicadas, 3 (6), pp. 227-235.

Plata, J.: «El municipio en la educación y en la formación de los deficientes ópticos». Revista de Psicología y Pedagogía Aplicadas, 3 (5), pp. 49-72.

Sánchez Jiménez, J.: «El niño bien dotado y los problemas que implica su asistencia especial». Revista de Psicología y Pedagogía Aplicadas, 3 (6), pp. 263-291.

Zaragozá, J.: «El censo escolar de Valencia». Revista de Psicología y Pedagogía Aplicadas, 3(5), pp. 9-30.

\section{3}

Bertrán, J.: «Errores principales que se cometen en las operaciones aritméticas fundamentales (I)». Revista de Psicología y Pedagogía Aplicadas, 4 (7-8), pp. 45-56.

Mallart, J.: «Selección escolar». Revista de Psicología y Pedagogía Aplicadas, 4 (7-8), pp. 27-44.

Selva, E. U.: «Participación del maestro en la orientación profesional escolar». Revista de Psicología y Pedagogía Aplicadas, 4 (7-8), pp. 15-25.

Zaragozá, J.: «Teoría de la medida del rendimiento escolar». Revista de Psicología y Pedagogía Aplicadas, 4 (7-8), pp. 57-64.

\section{4}

Bertrán, J.: «Errores principales que se cometen en las operaciones aritméticas fundamentales (y II)». Revista de Psicología y Pedagogía Aplicadas, 5 (9-10), pp. 93-116.

García Villegas, P.: «Profesiones femeninas de Servicio Social». Revista de Psicología y Pedagogía Aplicadas, 5 (9-10), pp. 93-116.

Germain, J.: «La psicología clínica y la escuela». Revista de Psicología y Pedagogía Aplicadas, 5 (910), pp. 11-37.

Mallart, J.: «Orientación escolar». Revista de Psicología y Pedagogía Aplicadas, 5 (9-10), pp. 77-92.

Sánchez Jiménez, J.: «Por una simbología universal del test Rorschach». Revista de Psicología y Pedagogía Aplicadas, 5 (9-10), pp. 39-54.

\section{5}

Delgado, H.: «Personalidad y delincuencia». Revista de Psicología y Pedagogía Aplicadas, 6 (11-12), pp. 11-25.

Gutiérrez Gómez, D.: «Papel del Psiquiatra psicotécnico en la Orientación profesional». Revista de Psicología y Pedagogía Aplicadas, 6 (11-12), pp. 43-61. 
Mallart, J.: «Práctica de la Orientación profesional en la escuela». Revista de Psicología y Pedagogía Aplicadas, 6 (11-12), pp. 27-41.

Zaragozá, J.: «Biotipo y profesión (I)». Revista de Psicología y Pedagogía Aplicadas, 6 (11-12), pp. 63-95.

1956

Alcamí, J.: «Morbilidad infantil y neurosis maternal y familiar». Revista de Psicología y Pedagogía Aplicadas, 7 (13-14), pp. 95-108.

Germain, J.: «Problemas y perspectivas en la Orientación profesional». Revista de Psicología y Pedagogía Aplicadas, 7 (13-14), pp. 13-70.

Mallart, J.: «Límites de la iniciación profesional». Revista de Psicología y Pedagogía Aplicadas, 7 (13-14), pp. 71-86.

Pertejo, J.: «Un aspecto de la profilaxis de los asociales: la carencia de amor maternal». Revista de Psicología y Pedagogía Aplicadas, 7 (13-14), pp. 87-94.

Plata, J.: «Ocupaciones profesionales para los ciegos». Revista de Psicología y Pedagogía Aplicadas, 7 (13-14), pp. 109-129.

Zaragozá, J.: «Biotipo y profesión (II)». Revista de Psicología y Pedagogía Aplicadas, 7 (13-14), pp. 131-155.

\section{Artículos y trabajos de laboratorio publicados en la revista de Psicología y Pedagogía Aplicadas (Segunda etapa: 1975-1981).}

1975

Artículos

Benavent, J. A.: «El análisis de tendencias en la interpretación de los fenómenos psicológicos de carácter evolutivo», 8 (16), pp. 75-88.

Marín Ibáñez, R.: «La enseñanza en equipo», 8 (15), pp. 7-32.

Sánchez Jiménez, J.: «Orientación psicopedagógica en educación maternal», 8 (16), pp. 9-28.

Zaragozá, J.: «Biotipo y Profesión (conclusión)», 8( 15), pp. 85-110.

\section{Trabajos de laboratorio}

Cerrudo, M. L. y Bañares, L. I.: «Test Thurstone-Mira de inteligencia general», 8 (16), pp. 133-142.

Gallardo, L., Lambies, A y Pérez Jordá, P.: «El test de aptitud espacial de las baldosas», 8 (16), pp. 111-120.

Olagüe, A.: «El test de Raven», 8 (15), pp. 117-128.

Rivas, F.: «Elaboración de un test numérico superior: El N. S.», 8 (16), 89-110.

Rivas, F. y Zaragozá, C.: «Modelo contrastado de predicción-seguimiento con alumnos de Ingeniería Superior de la Universidad Politécnica de Valencia», 8 (15), 129-142.

Torrijo, L., García Pascual, R., Vila, G. y Garrido, Ma . C. Estudio del test de memoria auditiva, 8( 16), pp. 121-132. 
1976

\section{Artículos}

Izquierdo, G.: «La inteligencia de los alumnos según el criterio de los profesores en relación con los tests y el rendimiento», 9 (17-18), pp. 41-66.

Lafuente, $\mathrm{M}^{\mathrm{a}}$. J.: «Extraversión y fluencia verbal», 9 (17-18), pp. 139-148.

McAlees, D.: «Explicaciones acerca del coeficiente intelectual», 9 (17-18), pp. 5-8.

Ontoria, A.: «Positividad y negatividad en la correlación de los rasgos de la personalidad», 9 (17-18), pp. 119-138.

Pereyra, M.: «El dibujo de la figura humana en la diagnosis de la obesidad infantil», 9 (17-18), pp. $27-40$.

\section{Trabajos de laboratorio}

Benlloch, C., Jiménez, C. y Marco, E.: «El factor verbal del test Thurstone-Mira», 9 (17-18), pp. 199204.

García Corrales, V. y Villanueva, M.: «Estudio de la Batería Factorial de Aptitudes (B.F.A.) en Valencia», 9 (17-18), pp. 149-180.

Rivas, F., Navarro, Mª L., Soria, Mª J. y Palau, C.: «Estudio de los intereses específicos dentro de la carrera de psicología y las influencias motivacionales de los estudiantes», 9 (17-18), pp. 181-198.

\section{7 (No se editó la revista)}

\section{8}

\section{Artículos}

Claudio, A.: «Estudio experimental sobre el rendimiento escolar de un grupo de alumnos en relación con la autonomía de la persona humana», 10 (19-20), pp. 95-104.

Ontoria, A.: «La adaptación en los alumnos de $5^{\circ}$ y $8^{\circ}$ de EGB», 10 (19-20), pp. 39-52.

Peiró y Gregori, S.: «Una experiencia de integración total para el segundo nivel de EGB», 10 (19-20), pp. 53-68.

\section{Trabajos de laboratorio}

Rivas, R. y Llosá, D.: «Cuestionario de intereses e influjos vocacionales: Un instrumento para el consejo orientador en la pubertad», 10 (19-20), pp. 117-126.

Rubio, L. V.: «El test de las matrices progresivas de Raven», 10 (19-20), pp. 105-116.

Villanueva, M., Soriano, F y Andreu, J. L.: «Ampliación del estudio de la B.F.A. de Manzione con estudiantes de EGB de Valencia», 10 (19-20), pp. 127-132. 
1979

\section{Artículos}

De la Rosa, B.: «Lineamientos básicos de la investigación socioeducativa», 11 (21-22), pp. 85-114. Sánchez Jiménez, J.: «Educación familiar y factores patogénicos», 11 (21-22), pp. 57-84.

\section{Trabajos de laboratorio}

Haba, Mª D. y Martín, R. Mª: «Test de atención», 11 (21-22), pp. 115-140.

Musitu, G., Pascual, J. y Ferrer, J.: «El marginado escolar. Influencia del status socio-económico», 11 (21-22), pp. 141-148.

\section{0}

\section{Artículos}

Ontoria, A.: «La educación y la influencia del sexo en la primera y la segunda etapa de la EGB», 12 (23-24), pp. 81-96.

Claudio, A.: «Experiencia terapéutica en un caso de autismo infantil», 12 (23-24), pp. 97-103.

Valcárcel, Ma . P.: «Validez del examen de acceso a la Universidad para mayores de veinticinco años», 12 (23-24), pp. 105-111.

López Torrijo, M. y Zaragozá, C.: «Influencia de las reuniones internacionales en la política hispanoamericana sobre Orientación (1934-1965)», 12 (23-24), pp. 113-120.

\section{Trabajos de laboratorio}

Galindo, J. V. et al.: «Estudio del test de comprensión de conocimientos básicos matemáticos de Buswell», 12 (23-24), pp. 137-150.

Musitu, G., Ferrer, J. y Pascual, J.: «El líder y el rendimiento escolar», 12 (23-24), pp. 121-135.

\section{1}

\section{Artículos}

Benavent, J. A. y Villalba, A.: «La organización del factor tiempo en los grupos de encuentro», 13 (25-26), pp. 9-25.

Andrés, A.: «Condicionamiento discriminativo de evitación en el aprendizaje de "Blaps Gigas" (Tenebrionidae)», 13 (25-26), pp. 27-47.

Aguilar, A.: «Rasgos de personalidad y comportamiento lector», 13 (25-26), pp. 49-65.

Miró, C. y Palacios, Mª C.: «Construcción y validación de una prueba objetiva», 13 (25-26), pp. 81105. 


\section{Trabajos de laboratorio}

Lafuente, $M^{\text {a }}$. J. y Valcárcel, M . P.: «Estudio sobre el test de Otis con estudiantes de EGB en Valencia», 13 (25-26), pp. 107-122.

Ramírez, V. M.: «Investigación sobre el test de conocimientos matemáticos básicos de Buswell», 13 (25-26), pp. 123-171.

Gutiérrez, L. J. y Vanegas, F. S.: «Normalización del test de los cuadrados de Rybakoff en estudiantes de $\mathrm{EGB} », 13$ (25-26), pp. 175-220.

Fecha de recepción: 01-09-03

Fecha de revisión:10-10-03

Fecha de aceptación: 27-10-03 